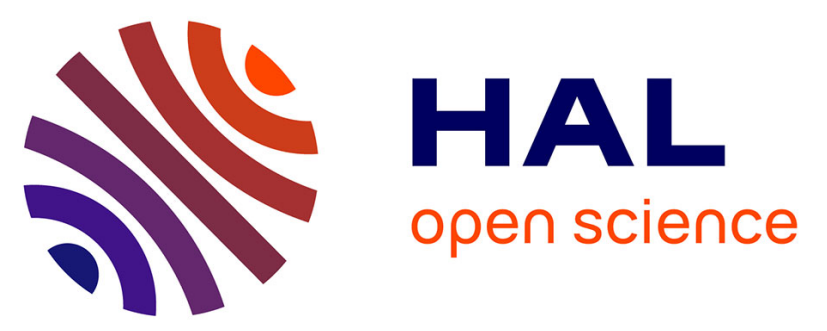

\title{
Comparison between subtalar joint quantitative kinematic 4-D CT parameters in healthy volunteers and patients with joint stiffness or chronic ankle instability:
}

\section{A preliminary study}

Pedro Augusto Gondim Teixeira, Anne-Sophie Formery, Gwenaël Balazuc, Guillaume Lux, Isabelle Loiret, Gabriela Hossu, Alain Blum

\section{To cite this version:}

Pedro Augusto Gondim Teixeira, Anne-Sophie Formery, Gwenaël Balazuc, Guillaume Lux, Isabelle Loiret, et al.. Comparison between subtalar joint quantitative kinematic 4-D CT parameters in healthy volunteers and patients with joint stiffness or chronic ankle instability: A preliminary study. European Journal of Radiology, 2019, 114, pp.76-84. 10.1016/j.ejrad.2019.03.001 . hal-03241105

\section{HAL Id: hal-03241105 \\ https://hal.univ-lorraine.fr/hal-03241105}

Submitted on 22 Oct 2021

HAL is a multi-disciplinary open access archive for the deposit and dissemination of scientific research documents, whether they are published or not. The documents may come from teaching and research institutions in France or abroad, or from public or private research centers.
L'archive ouverte pluridisciplinaire HAL, est destinée au dépôt et à la diffusion de documents scientifiques de niveau recherche, publiés ou non, émanant des établissements d'enseignement et de recherche français ou étrangers, des laboratoires publics ou privés.

\section{(ㄷ)(1) $\$$}

Distributed under a Creative Commons Attribution - NonCommerciall 4.0 International 


\title{
Comparison between Subtalar Joint Quantitative Kinematic 4-D CT
}

\section{Parameters in Healthy Volunteers and Patients with joint Stiffness}

\author{
or Chronic ankle instability: a preliminary study
}

\section{Original reserach}

Gondim Teixeira, Pedro Augusto MD, $\mathrm{PhD}^{1}$

Formery, Anne-Sophie MD ${ }^{1}$

Gwenaël, Balazuc MD ${ }^{1}$

Lux, Guillaume MD ${ }^{2}$

Isabelle, Loiret $\mathrm{MD}^{3}$

Hossu, Gabriela PhD ${ }^{4}$

Blum, Alain $\mathrm{MD}, \mathrm{PhD}^{1}$

1- Service D'imagerie Guilloz, Hôpital Central, CHU Nancy, F-54000, France. Tel: (+33) 383 851811

2- Radiolor, Clinique Louis Pasteur, Essey-lès-Nancy, F-54270, France. Tel : (+33) 38321 8080

3- Institut régional de médicine physique et réadaptation Louis Pierquin (IRR), 75 Boulevard Lobau 54042, Nancy, France. Tel : (+33) 383529700

4- Université de Lorraine, Inserm, IADI, F-54000 Nancy, France 


\section{Corresponding author:}

Pedro Augusto, Gondim Teixeira - Service D'imagerie Guilloz, Hôpital Central, CHU Nancy, F54000, France. Tel: (+33) 3838518 11, Fax : (+33) 383859725

e-mail : ped_gt@hotmail.com 


\title{
Comparison between Subtalar Joint Quantitative Kinematic 4-D CT
}

\section{Parameters in Healthy Volunteers and Patients with joint Stiffness}

\author{
or Chronic ankle instability: a preliminary study
}

\section{Original research}

\begin{abstract}
Objective: to compare quantitative kinematic 4-D CT parameters between healthy volunteers and pathologic patients (joint stiffness or chronic ankle instability).
\end{abstract}

Methods: Thirteen healthy volunteers without previous ankle trauma, 18 patients with clinical subtalar joint stiffness and 10 patients with chronic ankle instability diagnosed based on clinical and imaging findings were prospectively evaluated with 4-D CT. This study was approved by the local ethics committee and all patients signed an informed consent. The subtalar joint was evaluated during a prono-supination cycle. Two angles and two distances between the talus and the calcaneus were measured semi-automatically and independently by two readers. Measurement variations were compared in these three different groups.

Results: There were statistically significant differences between axial and coronal talocalcaneal angles of healthy volunteers and patients with joint stiffness $(p<0.0001)$. The best sensitivities and specificities for the identification of subtalar joint stiffness were 92$100 \%$ and $74-94 \%$. Mean and maximal posterior calcaneal facet uncovering were significantly lower in patients with chronic ankle instability patients compared to healthy volunteers $(p<0.006)$ with sensitivities and specificities of $92-95 \%$ and $80-92 \%$ respectively. 
Conclusion: Quantitative analysis in 4D CT can provide an objective criteria for the differentiation between healthy volunteers and patients with subtalar joint stiffness and chronic ankle instability.

\section{Keywords}

Computed tomography; subtalar joint; dynamic; chronic ankle instability; joint stiffness. 


\section{Introduction}

Traumatic injury is a frequent cause of subtalar joint pathology. Complex and displaced calcaneal fractures can lead to subtalar joint stiffness, pain and disability related to secondary subtalar degenerative joint disease despite appropriate surgical fracture reduction $[1,2]$. Subtalar joint arthrodesis is indicated in cases of persistent pain upon weight bearing, walking or performing physical activity after calcaneal fractures [1]. There is little information available on the imaging evaluation of the degree of joint amplitude loss and on the dynamic evaluation of arthrodesis effectiveness.

Subtalar joint injury is relatively frequent after ankle trauma ( $25 \%$ of ankle sprains) [3]. Repeated lateral ankle sprains can lead in about a third of cases to persistent pain and functional deficits, a condition termed chronic ankle instability [4]. Chronic ankle instability is most frequently associated with injuries to the calcaneofibular ligament (ankle), extensor retinaculum and to the cervical ligament (subtalar joint) [5]. The identification and assessment of the subtalar component of chronic ankle instability is of relevance as patient with suspected subtalar joint injury require specific treatment (most frequently physiotherapy, muscle strengthening and immobilization) [5]. Although clinical stress testing (internal rotation and varus with the ankle fixed in extension) and visual weight bearing evaluation of the hind foot can suggest subtalar instability, the clinical assessment of the subtalar joint remains difficult because this joint has a complex anatomy and biomechanics [3]. Additionally, there is no recognized way to objectively assess treatment response in patients with chronic ankle instability and in this context non-invasive quantitative analysis of subtalar joint motion could prove helpful. 
Imaging methods allowing a dynamic evaluation of the subtalar joint amplitude during motion could improve diagnostic accuracy and provide objective criteria for patient followup. Dynamic 4-D CT is performed by acquiring various low-dose sequential volumes with high temporal resolution of a moving joint and has been used for the dynamic evaluation of various joints such as wrist, hip, elbow and shoulder girdle [6-11]. In a recent cadaveric and healthy volunteer study Gondim Teixeira et al. showed that quantitative dynamic 4-D CT could performed on the subtalar joint suggesting a potential role of this technique in assessing joint instability and stiffness [12]. Increases in joint amplitudes after section of subtalar ligaments in cadavers were reported, confirming the potential of this technique in clinical practice. Recently, the use of quantitative 4-D CT has been demonstrated in the wrist, providing quantitative criteria for the differentiation of patient with and without dissociative scapholunate instability $[13,14]$. In a context that CT is frequently used for the evaluation of patients with hind foot trauma for fracture detection and consolidation assessment, 4-D CT could be useful to ascertain the biomechanical impact of the injuries sustained [15-17].

We hypothesize that healthy volunteers, patients with clinical chronic ankle instability and subtalar joint stiffness can be differentiated based on quantitative assessment of subtalar joint amplitude with 4-D CT, which could provide additional diagnostic criteria for diagnosis and patient follow-up. Thus, four previously established 4-D CT derived parameters were compared in these three groups [12]. The value distribution of two angles, axial talocalcaneal (ATCA) used to assess subtalar internal-external rotation and coronal talocalcaneal (CTCA) used to assess talar tilt angles as well as two distances, medial facet gap (MFG) used to assess subtalar eversion laxity and posterior calcaneal facet uncovering (PCFU) used to 
assess the congruence of the posterior subtalar joint congruence were evaluated during a subtalar joint prono-supination cycle $[3,18,19]$.

\section{Material and methods}

Study population

From November 2011 to December 2012, 38 consecutive patients were prospectively included in this ethics committee approved study. All patients were over 18 years-old, signed informed consent and underwent kinematic 4-D CT of the subtalar joint. All patients had been referred by orthopedic surgeons and rheumatologists for the evaluation of ankle pain associated with either subtalar joint stiffness or chronic ankle instability. Patients with metallic implants, inflammatory arthritis and congenital subtalar joint conditions were not included in this study. In addition, 17 healthy volunteers with no previous ankle trauma and a normal physical examination of the ankle who underwent kinematic 4-D CT of the subtalar joint were included. Physical examination was performed in all volunteers prior to CT acquisitions by a senior radiologist with at least five years of clinical experience. The evaluation of joint hyperlaxity was based on the Beighton score. One volunteer, despite an initial negative clinical history, had periosteal irregularities in the fibular insertion of the anterior talofibular ligament, which could be related to anterior ankle trauma. Two volunteers failed to perform a homogeneous prono-supination motion during the CT scan and were excluded. Two volunteers who presented clinical signs of hyperlaxity with a Beighton score higher than two were also excluded. Since one of the volunteers underwent bilateral 4-D kinematic CT, 13 ankles composed the healthy volunteer group. 
Eighteen patients with signs of stiffness were also included. Patients with post-traumatic clinical limitation of the subtalar joint amplitude of motion in plantarflexion, dorsiflexion and inversion compared to the uninjured contralateral side were considered positive for subtalar joint stiffness.

Twenty patients with signs or clinical history of ankle trauma with persistent pain (at least one month) were evaluated. Chronic ankle instability diagnosis was confirmed when these patients had at least one injured ankle ligament on MRI or CT arthrography. Ligament sprains on MRI (T2 signal hyperintensity and thickening without clear loss in continuity), partial tears (partial loss in ligament continuity) and full tears (complete loss in ligament fiber continuity) on MRI or CT arthrography were evaluated. The lateral and medial collateral ligament complexes, as well as the cervical and interosseous subtalar ligaments were considered in the analysis. Among these patients, 10 were excluded due to unclear ankle trauma history, previous surgery, no ligament anomalies found on imaging, no imaging available and failure to perform a homogeneous prono-supination motion during the CT scan (one patient). Thus the chronic ankle instability group was composed of 10 ankles. Table 1 shows the clinical characteristics and imaging findings these patients.

\section{Image acquisition}

Acquisitions were performed using a wide detector CT scanner with 320-detector rows (Aquilion ONE, Canon Medical Systems, Otawara, Japan) using intermittent sequential mode (no table feed), $24 \mathrm{~cm}$ FOV, 512x512 matrix, $0.5 \mathrm{~mm}$ slice thickness, tube rotation 0.35 seconds. Tube output was $80 \mathrm{kVp}$ and $65 \mathrm{mAs}$ (22 mA effective). Z-axis coverage varied from $8-12 \mathrm{~cm}$ depending on the patient morphology. Images were reconstructed with half reconstruction using bone and soft-tissue kernels. Adaptive iterative reconstruction (AIDR 
3D, Canon Medical Systems, Otawara, Japan) in the standard level was used for all acquisitions.

The images were acquired during a prono-supination cycle of the subtalar joint. All acquisitions were unilateral. The patient was placed supine on the scanner table. The examination began in the maximum pronation position and ended when the foot returned to the starting point after supination, lasting $10-15$ seconds. An elastic band was used to guide the movement (Fig. 1). A training session was conducted before CT acquisitions to ensure a controlled and homogeneous movement of patients. The volume CT dose index $\left(C T D I_{\text {vol }}\right)$ and the dose length product (DLP) of patient acquisitions were estimated based on a phantom $16 \mathrm{~cm}$ in diameter. Effective doses were calculated using a conversion coefficient of $0.0002[20]$.

Image analysis and post processing

All measurements in pathologic patients were performed independently by two readers with four (A-S.F.) and two (G.B.) years of experience in musculoskeletal radiology. Reference measures in normal volunteers were obtained by a third reader (P.T.) with 12 years of experience in musculoskeletal radiology. This reader also evaluated the MRI and CT arthrography studies in patients with suspected chronic ankle instability performed with a conventional acquisition protocol. Images were post-processed in a display console workstation V7.0 (Canon Medical Systems, Otawara, Japan) using the 4-D Ortho clinical application tool. Image post-processing was as follows. All the volumes of a kinematic acquisition were loaded and multiplanar and 3-D volume rendered images were displayed. The examiner then placed a seed point on the medullary cavity of the talus and the 
registration algorithm was executed. After registration, all motion was displayed with respect to the talus, which remained static, serving as a reference for plane selection. The axial plane was defined by the long axis of the talus, coronal plane was defined by the bimalleolar axis and the sagittal plane was defined by the long axis of the tibia. Then, the examiner placed two reference markers for distance measurements and four for angular measurements over the cortical bone on the multiplanar reformats in a single acquisition volume. These markers were plotted automatically by the software to all acquisition volumes. Four different measurements were performed to describe the talocalcaneal relationship during prono-supination according to the previous work of Gondim Teixeira et al. [12] (Fig. 2):

- The coronal talocalcaneal angle (CTCA). The reference markers were place in a bi-malleolar coronal plane with the ankle in neutral position. A line was constructed by placing two markers at the medial and lateral extremes of the talar dome. A second line was constructed in the calcaneus by placing one marker at the most lateral point of the sustentaculum tali and another on deepest portion of Gissagne's angle. The angle formed between these lines was measured $[18,21,22]$.

- The axial talocalcaneal angle (ATCA). The reference markers were placed in the axial plane passing through the long axis of the talus. At the level of the talus, the slice depicting the summit of the talar head and on the postero-medial process of the talus (Stieda process) was selected. A line was constructed by placing one marker on each of these two points. At the level of the calcaneus, two markers were placed in a slice depicting the middle of the calcaneocuboid articular facet and another on midpoint of the posterior calcaneal surface. A 
line between two markers was constructed. The angle formed between these lines was measured [3].

- Medial facet gap (MFG). The reference markers were place with the ankle in neutral position. The slice depicting the most lateral point on the medial facet of the talus without partial volume effect was selected. In this slice, markers were placed on the medial facets of the talus and the calcaneus so that the shortest distance between these two bones was measured [22].

- Posterior calcaneal facet uncovering (PCFU). This measurement was performed in a sagittal plane with the ankle in neutral position was selected. The reference markers were placed using the volume depicting full ankle supination. The markers were placed at the extremes of the largest distance between the center of Gissagne's angle (identified based on bone contours and the location of the nutrient foramina) and the anterior border of the posterior facet of the talus [19].

Once the land marks for marker placement were established, the post-processing time per data set including data loading, bone locking and performing the four measurements described lasted roughly 15 minutes.

\section{Statistics}

Statistical analysis was carried out using the R software (Version 3.0.0, The R Foundation for Statistical Computing, Vienna, Austria) with the pROC and IRR packages [23]. The changes in ratio and absolute values of the measures acquired were compared. Ratios were calculated by dividing the values obtained by the maximum pronation value. Ratios were calculated because the direct comparison of absolute values is imprecise due to technical factors, such 
as minor variations in reference position and differences in bone size between subjects. For each subject, four measurements were performed two angles (CTCA and ATCA) and two distances (MFG and PCFU). For each measurement a series of ratios was available (e.g. one value for each phase of the dynamic acquisition). For each measurement series acquired, the mean, standard deviation (SD), coefficient of variation (CV), maximal values, minimal values and amplitude variation were analyzed to assess subtalar joint motion during pronosupination. Intraclass correlation coefficients for the ratios of each of the parameters evaluated were calculated to assess measurement inter-observer variability. ICC values of 00.20 were considered slight agreement, $0.21-0.40$ fair, $0.41-0.60$ moderate, $0.61-0.80$ substantial, and 0.81-1 was accepted as excellent agreement. The results were presented on average \pm standard deviation. The Wilcoxon test was used to assess the statistical significance of the parameters evaluated between the subgroups evaluated (healthy volunteers versus patients with subtalar joint stiffness and healthy volunteers versus patient with chronic ankle instability). A Bonferroni correction was applied to account for multiple comparisons and a $P$ value $<0.003$ was considered as the threshold for statistical significance. ROC analysis was performed for all parameters (mean, SD, CV and amplitude) that showed a statistically significant differences between volunteers/patients with joint stiffness and volunteers/patients with joint instability.

\section{Results}

There were four men and eight women in the healthy volunteer group (Male/female ratio = 0.5 ) with a mean age of $32.7 \pm 7.2$ years, ranging from 24 to 50 years. Mean values of CTCA, ATCA, MFG and PCFU in healthy volunteers were $23.6 \pm 8.3^{\circ}, 14.8 \pm 6.6^{\circ}, 7.1 \pm 4.4 \mathrm{~mm}$ and 
$16.3 \pm 1.8 \mathrm{~mm}$ respectively. Among healthy volunteers $\mathrm{CV}$ values were higher in angular than in distance parameters ( $36 \%$ and $45.8 \%$ for CTCA and ATCA respectively compared to $20 \%$ and $11.4 \%$ for MFG and PCFU respectively) (Fig. 3).

The interobserver variability of the measurements performed in the patients evaluated was considered excellent with ICC values of 0.92 in patients with chronic ankle instability. In this subgroup angular measurement presented excellent while distance measurement presented substantial interobserver variabilities (ICC $=0.94$ for ATCA/CTCA and 0.72 and 0.75 for MFG and PCFU respectively). The interobserver variability was also considered excellent with ICC values of 0.81 in patients with joint stiffness. In this subgroup CTCA and PCFU presented an excellent interobserver variability (0.93 and 0.9 respectively) while it was substantial with ATCA (0.79) and moderate with MFG (0.44). The mean CTDI volume, DLP and effective dose of the studies performed were $20.6 \pm 5.6 \mathrm{mGy}, 168.1 \pm 43.7 \mathrm{mGy} . \mathrm{cm}$ and $0.067 \pm 0.01 \mathrm{mSv}$, respectively.

\section{Patients with subtalar joint stiffness}

There were eight men and ten women among patients with subtalar joint stiffness (Male/female ratio $=0.8)$. The age in this group was higher than that of the healthy volunteers with a mean age of $52.8 \pm 11.8$ years ranging from 26 to 71 years. Mean values of all parameters evaluated in this subgroup and the $P$ values with respect to healthy volunteer data are presented in table 2 . Compared to normal volunteers there was a general tendency towards reduction in the parameters evaluated in patients with subtalar joint stiffness. With both angles and MFG, amplitude and CV values had a statistically significant difference between volunteers and patients with subtalar joint stiffness for both readers $(P$ $<0.0011$ )(Fig. 4)(Supplementary material 1). If the CV and amplitude values of CTCA, ATCA 
and MFG were considered there was a 54-67\% value reduction in patients with subtalar joint stiffness with respect to healthy volunteers. As expected, maximum values of were also lower in patients with joint stiffness compared to healthy volunteers, with the exception of MFG values for reader 1 . However, these differences were only significant with PCFU.

After ROC analysis, for both readers, PCFU yielded the best sensitivity for subtalar joint stiffness identification. Sensitivity was $92 \%$ with PCFU maximal values (cut off $17 \mathrm{~mm}$ ) and CV (6\% cut off) and of a $100 \%$ using PCFU amplitude $(1.6 \mathrm{~mm})$. Both angular measurements (CTCA and ATCA) yielded the best specificity values for both readers varying from $74-94 \%$. CTCA CV yielded the highest sensitivity for both readers (85-94\% with a $28 \%$ cut off). The diagnostic performance of maximum values, CV and variation amplitude for all angles and distances measured is presented on Table 3.

\section{Patients with Chronic ankle instability}

There were four men and six women among patients with chronic ankle instability (Male/female ratio $=0.66)$ with a similar age then healthy volunteers mean age of $32.6 \pm 9$ years ranging from 21 to 47 years. There are no records of surgical treatment following imaging for these patients in our database. Mean values of all parameters evaluated in this subgroup and the $P$ values relative to healthy volunteer data are presented in table 4 . The only measurement that yielded statistically significant differences with respect to the healthy volunteer group for both readers was PCFU maximum values. There was a $25 \%-44 \%$ decrease in maximal values PCFU for readers 1 and 2 respectively $(P$ varied from $<0.001)$ in patients with chronic ankle instability. The PCFU CV values increased in patients with chronic ankle instability compared to healthy volunteers for both readers (29\%-48\% increase for 
readers 1 and 2 respectively)(Fig. 5)(Supplementary material 2). These differences, however, were not statistically significant ( $P=0.31$ and 0.006 for readers 1 and 2 respectively). With ROC analysis performed with PCFU derived parameters, maximal values yielded the best sensitivity $92-95 \%$ and the best specificities $80-92 \%$ (cut off $17 \mathrm{~mm}$ ) for the identification of chronic ankle instability.

For reader one, mean and maximum MFG were higher ( $23.4 \%$ and $16.6 \%$ respectively) in patients with chronic ankle instability compared to healthy volunteers. Reader two data showed a slight decrease in mean and maximum MFG values in chronic ankle instability (5.3\% and $-5.7 \%)$. These differences, however, were not statistically significant $(P>0.016)$.

\section{Discussion}

The differentiation between healthy volunteers and patients with subtalar joint stiffness and chronic ankle instability was possible with kinematic 4-D CT. There were significant changes in all parameters evaluated (ATCA, CTCA, MFG and PCFU) between volunteers and patients with joint stiffness ( $P$ varying from $<0.0001$ to 0.0015$)$. In patients with chronic ankle instability PCFU values were significantly different than those of healthy volunteers ( $P$ values varying from $<0.0001$ to 0.001 ). Measurement ratio interobserver reproducibility in pathologic patients was considered to be excellent (ICC >0.81) which is a considerable advantage of this semi-automatic method compared to manual measurements [24]. The interobserver reproducibility of each individual measurement was considered substantial to excellent (ICC varying from 0.72 to 0.94 ) with the exception of MFG in patients with joint stiffness which was considered moderate $(I C C=0.4)$. This finding could be related to difficulties in marker placement in patients with calcaneal morphologic changes secondary to fractures. Moreover, the effective dose was lower than $<0.1 \mathrm{mSv}$. These results indicate 
that kinematic 4-D CT can have a potential role in the evaluation of subtalar joint pathology providing means for an objective joint amplitude assessment which could improve the accuracy in identifying subtalar involvement in patients with chronic ankle instability and a more precise assessment of subtalar joint stiffness.

In patients with subtalar joint stiffness, there was a significant reduction in the variation amplitude of all measurements performed $(p<0.02)$. CTCA, ATCA and MFG were the best measurements for the identification of subtalar joint stiffness with a reduction of more than $50 \%$ of the $\mathrm{CV}$ and variation amplitude with respect to healthy volunteers. The performance of kinematic 4-D CT for the identification of patients with clinical joint stiffness was also good. PCFU related parameters yielded the best sensitivities (92-100\%) and the best specificity was reached when CV and variation amplitude of angular measurements (CTCA and ATCA) were assessed (72-94\%). Thus, Kinematic 4-D CT could be an interesting option to objectively confirm and grade subtalar joint stiffness. This method could also be interesting for the evaluation of the effectiveness of orthopedic constraint devices and surgical arthrodesis on subtalar joint motion by detecting and quantifying residual micro motion. Although PCFU analysis yielded statistically significant results for the differentiation between healthy volunteers and patients with chronic ankle instability $(p<0.001)$ with high sensitivity and specificity values ( $92 \%$ and $95 \%$ respectively). This data should be interpreted with caution. There was a reduction in mean and maximal PCFU in this patient group compared to healthy volunteers, which could seem paradoxical. Patients with chronic ankle instability usually present with pain and proprioception issues with apprehension during subtalar joint motion $[25,26]$. This could explain at least partially the reduction in PCFU values compared to normal volunteers. Although statistically significant for reader 2 only $(P=0.006)$, both 
readers identified an increase in the CV of PCFU in patients with instability compared to healthy volunteers (24\%-48\%). These findings should be confirmed by larger studies but may suggest a change in the respective motion between the talus and calcaneus in patients with joint instability even if maximal joint amplitudes remain normal or decreased, a phenomenon similar to carpal bone hysteresis [27].

In a previous cadaveric study on subtalar joint 4-D CT it was suggested that MFG assessment would be better suitable for the evaluation of subtalar joint instability as this parameter presented the highest increase before and after resection of subtalar joint ligaments (cervical and interosseous)[12]. In the present study, the mean and maximum MFG were higher in patients with chronic ankle instability compared to normal volunteers only for one of the readers and these differences were not statistically significant $(P>0.015)$. This could be at least partially explained by the fact that the injuries sustained by the evaluated patients were likely less severe than the full tearing of the cervical and interosseous ligaments created in the prior cadaveric study. Additionally, the small number of patients in the chronic instability group could have also contributed to this discrepancy.

Various limitations of this study must be acknowledged. The subtalar joint has not been evaluated under weight-bearing conditions. This could lead to an underestimation of the diagnostic performance of kinematic 4-D CT for the identification of subtalar joint pathology, especially in patients with chronic ankle instability. A device allowing kinematic ankle imaging under stress is currently being developed. Such a device is could increase the diagnostic performance of this method [28]. Another limitation is the size of the study population in general but particularly in the chronic ankle instability group. As the nonsurgical confirmation of chronic ankle instability is challenging, we chose to use strict clinical 
and imaging criteria, which led to a $50 \%$ reduction of the initial patient population. Thus, studies with larger patient groups are needed to confirm the presented findings. Some patients did not succeed in performing adequate subtalar joint prono-supination which may suggest the need for a more aggressive guidance of patient motion. The ligament lesions visualized on MRI and CT arthrography were not surgically confirmed as the patients included were not managed surgically. This evaluation, however, was performed by an experienced musculoskeletal radiologist based on previously described imaging criteria. Further studies are also necessary to compare the diagnostic performance of stress MRI and kinematic 4-D CT.

In conclusion, Kinematic 4-D CT is an objective and reproducible tool for the evaluation of in vivo subtalar joint motion. This method could provide objective criteria subtalar joint assessment with a potential impact on patient management as there were significant changes in the quantitative parameters evaluated in patients with subtalar joint stiffness and patients with chronic ankle instability with respect to healthy volunteers with high sensitivities and specificities. Although further studies in larger patient populations and with weight bearing simulation are needed, particularly in patients with chronic ankle instability, kinematic 4-D CT shows potential for the objective evaluation of patients with subtalar joint pathology. 


\section{Bibliography}

[1] G. Romeo, N. Martinelli, C. Bonifacini, A. Bianchi, E. Sartorelli, F. Malerba, Recreational Sports Activities After Calcaneal Fractures and Subsequent Subtalar Joint Arthrodesis, J. Foot Ankle Surg. Off. Publ. Am. Coll. Foot Ankle Surg. 54 (2015) 1057-1061. doi:10.1053/j.jfas.2015.05.003.

[2] J.L. Holm, S.E. Laxson, J.M. Schuberth, Primary subtalar joint arthrodesis for comminuted fractures of the calcaneus, J. Foot Ankle Surg. Off. Publ. Am. Coll. Foot Ankle Surg. 54 (2015) 61-65. doi:10.1053/j.jfas.2014.07.013.

[3] S. Weindel, R. Schmidt, S. Rammelt, L. Claes, A. v Campe, S. Rein, Subtalar instability: a biomechanical cadaver study, Arch. Orthop. Trauma Surg. 130 (2010) 313-319. doi:10.1007/s00402-008-0743-2.

[4] T. Mittlmeier, A. Wichelhaus, Subtalar joint instability, Eur. J. Trauma Emerg. Surg. Off. Publ. Eur. Trauma Soc. 41 (2015) 623-629. doi:10.1007/s00068-015-0588-7.

[5] M.J. Hentges, M.S. Lee, Chronic ankle and subtalar joint instability in the athlete, Clin. Podiatr. Med. Surg. 28 (2011) 87-104. doi:10.1016/j.cpm.2010.10.001.

[6] S. Leng, K. Zhao, M. Qu, K.-N. An, R. Berger, C.H. McCollough, Dynamic CT technique for assessment of wrist joint instabilities, Med. Phys. 38 Suppl 1 (2011) S50. doi:10.1118/1.3577759.

[7] Y. Edirisinghe, J.M. Troupis, M. Patel, J. Smith, M. Crossett, Dynamic motion analysis of dart throwers motion visualized through computerized tomography and calculation of the axis of rotation, J. Hand Surg. Eur. Vol. 39 (2014) 364-372. doi:10.1177/1753193413508709.

[8] S. Demehri, N. Hafezi-Nejad, U. Thakur, J.N. Morelli, S.D. Lifchez, K.R. Means, J.T. Shores, Evaluation of pisotriquetral motion pattern using four-dimensional CT: initial 
clinical experience in asymptomatic wrists, Clin. Radiol. (2015).

doi:10.1016/j.crad.2015.07.007.

[9] G.I. Wassilew, V. Janz, M.O. Heller, S. Tohtz, P. Rogalla, P. Hein, C. Perka, Real time visualization of femoroacetabular impingement and subluxation using 320 -slice computed tomography, J. Orthop. Res. Off. Publ. Orthop. Res. Soc. 31 (2013) 275-281. doi:10.1002/jor.22224.

[10] Y.P. Goh, K.K. Lau, Using the 320-Multidetector Computed Tomography scanner for four-dimensional functional assessment of the elbow joint, Am. J. Orthop. Belle Mead NJ. 41 (2012) E20-24.

[11] P.A. Gondim Teixeira, A. Gervaise, M. Louis, S. Lecocq, A. Raymond, S. Aptel, A. Blum, Musculoskeletal wide detector CT: principles, techniques and applications in clinical practice and research, Eur. J. Radiol. 84 (2015) 892-900. doi:10.1016/j.ejrad.2014.12.033.

[12] P.A. Gondim Teixeira, A.-S. Formery, A. Jacquot, G. Lux, I. Loiret, M. Perez, A. Blum, Quantitative Analysis of Subtalar Joint Motion With 4D CT: Proof of Concept With Cadaveric and Healthy Subject Evaluation, AJR Am. J. Roentgenol. 208 (2017) 150-158. doi:10.2214/AJR.16.16434.

[13] W. Abou Arab, A. Rauch, M.B. Chawki, F. Dap, G. Dautel, A. Blum, P.A. Gondim Teixeira, Scapholunate instability: improved detection with semi-automated kinematic CT analysis during stress maneuvers, Eur. Radiol. (2018). doi:10.1007/s00330-018-5430-2.

[14] A. Rauch, W.A. Arab, F. Dap, G. Dautel, A. Blum, P.A. Gondim Teixeira, Four-dimensional CT Analysis of Wrist Kinematics during Radioulnar Deviation, Radiology. (2018) 180640. doi:10.1148/radiol.2018180640. 
[15] F. Berger, L.C. Ebert, R.A. Kubik-Huch, K. Eid, M.J. Thali, T. Niemann, Application of Cinematic Rendering in Clinical Routine CT Examination of Ankle Sprains, AJR Am. J. Roentgenol. 211 (2018) 887-890. doi:10.2214/AJR.17.18630.

[16] K. Badillo, J.A. Pacheco, S.O. Padua, A.A. Gomez, E. Colon, J.A. Vidal, Multidetector CT evaluation of calcaneal fractures, Radiogr. Rev. Publ. Radiol. Soc. N. Am. Inc. 31 (2011) 81-92. doi:10.1148/rg.311105036.

[17] Y. Chen, K. Zhang, M. Qiang, H. Li, H. Dai, Comparison of plain radiography and CT in postoperative evaluation of ankle fractures, Clin. Radiol. 70 (2015) e74-82. doi:10.1016/j.crad.2015.04.011.

[18] C.J. Seebauer, H.J. Bail, J.C. Rump, B. Hamm, T. Walter, U.K.M. Teichgräber, Ankle laxity: stress investigation under MRI control, AJR Am. J. Roentgenol. 201 (2013) 496-504. doi:10.2214/AJR.12.8553.

[19] T. Kato, The diagnosis and treatment of instability of the subtalar joint, J. Bone Joint Surg. Br. 77 (1995) 400-406.

[20] N. Saltybaeva, M.E. Jafari, M. Hupfer, W.A. Kalender, Estimates of effective dose for CT scans of the lower extremities, Radiology. 273 (2014) 153-159. doi:10.1148/radiol.14132903.

[21] J.W. Louwerens, A.Z. Ginai, B. van Linge, C.J. Snijders, Stress radiography of the talocrural and subtalar joints, Foot Ankle Int. 16 (1995) 148-155.

[22] M.L. Cordova, J.M. Sefton, T.J. Hubbard, Mechanical joint laxity associated with chronic ankle instability: a systematic review, Sports Health. 2 (2010) 452-459. doi:10.1177/1941738110382392. 
[23] X. Robin, N. Turck, A. Hainard, N. Tiberti, F. Lisacek, J.-C. Sanchez, M. Müller, pROC: an open-source package for R and S+ to analyze and compare ROC curves, BMC Bioinformatics. 12 (2011) 77. doi:10.1186/1471-2105-12-77.

[24] D. Forsberg, M. Lindblom, P. Quick, H. Gauffin, Quantitative analysis of the patellofemoral motion pattern using semi-automatic processing of 4D CT data, Int. J. Comput. Assist. Radiol. Surg. 11 (2016) 1731-1741. doi:10.1007/s11548-016-1357-8.

[25] G. Pisani, Chronic laxity of the subtalar joint, Orthopedics. 19 (1996) 431-437.

[26] T. Vaseenon, Y. Gao, P. Phisitkul, Comparison of two manual tests for ankle laxity due to rupture of the lateral ankle ligaments, lowa Orthop. J. 32 (2012) 9-16.

[27] S. Berdia, W.H. Short, F.W. Werner, J.K. Green, M. Panjabi, The hysteresis effect in carpal kinematics, J. Hand Surg. 31 (2006) 594-600. doi:10.1016/j.jhsa.2005.12.028.

[28] P.A. Gondim Teixeira, A. Aich, A.-S. Formery, W. Abou Arab, A. Rauch, I. Loiret, A. Blum, State-of-the-Art Subtalar Joint Kinematic and Stress Imaging with Emphasis on 4-D CT: Where Do We Stand and Where Are We Going, Médecine Chir. Pied. (2018). doi:10.3166/mcp-2018-0016. 


\section{Tables}

Table 1 - Clinical and demographic characteristics of patients in the chronic ankle instability group.

\begin{tabular}{|c|c|c|c|c|}
\hline Patients & Sex & Age & Clinical findings & Imaging findings \\
\hline 1 & $\mathrm{~F}$ & 42 & $\begin{array}{l}\text { Ankle sprain } 7 \text { months prior, } \\
\text { instability sensation and pain }\end{array}$ & ATFL and CFL sprain on MRI \\
\hline 2 & $\mathrm{~F}$ & 21 & $\begin{array}{l}\text { Prior history of severe ankle trauma, } \\
\text { persistant pain }\end{array}$ & ATFL full tear and DL sprain on MRI \\
\hline 3 & $\mathrm{~F}$ & 47 & $\begin{array}{c}\text { Severe ankle trauma } 2 \text { year prior, } \\
\text { persistant pain }\end{array}$ & ATFL full tear on CT arthrography \\
\hline 4 & M & 33 & $\begin{array}{c}\text { Multiple ankle sprains, persistant } \\
\text { pain }\end{array}$ & ATFL full tear on MRI \\
\hline 5 & M & 34 & $\begin{array}{l}\text { Multiple ankle sprains, recent acute } \\
\text { sprain }\end{array}$ & $\begin{array}{l}\text { ATFL full tear, CFL sprain, DL partial tear, } \\
\text { cervical ligament sprain }\end{array}$ \\
\hline 6 & M & 21 & $\begin{array}{c}\text { Multiple prior sprains, persistant } \\
\text { pain }\end{array}$ & $\begin{array}{l}\text { ATFL partial tear, Anterior tibiofibular } \\
\text { ligament sprain on MRI }\end{array}$ \\
\hline 7 & $\mathrm{~F}$ & 42 & $\begin{array}{l}\text { Ankle sprain } 6 \text { months prior, } \\
\text { persistant pain }\end{array}$ & ATFL full tear, CFL and DL sprain on MRI \\
\hline 8 & M & 28 & $\begin{array}{l}\text { Ankle sprain } 6 \text { months prior, } \\
\text { instability sensation and pain }\end{array}$ & $\begin{array}{c}\text { ATFL full tear, DL partial tear, posterior } \\
\text { tibiofibular ligament sprain on CT } \\
\text { arthrography }\end{array}$ \\
\hline 9 & $\mathrm{~F}$ & 25 & Multiple sprains, persistant pain & ATFL full tear on MRI \\
\hline 10 & $\mathrm{~F}$ & 31 & $\begin{array}{l}\text { Multiple prior ankle sprains, recent } \\
\text { acute sprain }\end{array}$ & ATFL and CFL full tear on MRI \\
\hline
\end{tabular}

ATFL = Anterior talofibular ligament

$\mathrm{CFL}=$ Calcaneofibular ligament

$\mathrm{DL}=$ Deltoid ligament 
Table 2 - Mean values of all parameters evaluated in patients with subtalar joint stiffness and variation with respect to healthy volunteers.

\begin{tabular}{|c|c|c|c|c|c|c|c|c|c|c|c|c|c|}
\cline { 2 - 13 } \multicolumn{1}{c|}{ Reader 1 } & Mean & Sdev & Variation & $P$ Value & CV & Variation & $P$ Value & Amplitude & Variation & $P$ Value & Max & Variation & $P$ Value \\
\hline CTCA $\left(^{\circ}\right)$ & 20.0 & 3.1 & $-17.9 \%$ & 0.2935 & $15.5 \%$ & $-57.0 \%$ & $0.0002^{*}$ & 8.2 & $-61.6 \%$ & $0.0002^{*}$ & 24.4 & $-32.6 \%$ & 0.0065 \\
\hline ATCA $\left(^{\circ}\right)$ & 12.8 & 2.4 & $-15.1 \%$ & 0.1697 & $19.0 \%$ & $-58.6 \%$ & $0.0003^{*}$ & 6.4 & $-62.7 \%$ & $0.0000^{*}$ & 15.9 & $-27.3 \%$ & 0.0095 \\
\hline MFG (mm) & 8.3 & 0.6 & $14.8 \%$ & 0.0464 & $6.8 \%$ & $-66.1 \%$ & $0.0001^{*}$ & 1.6 & $-57.7 \%$ & $0.0015 *$ & 9.2 & $5.6 \%$ & 0.9520 \\
\hline PCFU (mm) & 12.4 & 1.0 & $-31.8 \%$ & $0.0001 *$ & $8.1 \%$ & $-28.8 \%$ & 0.0965 & 2.6 & $-44.7 \%$ & 0.0181 & 13.8 & $-27.4 \%$ & $0.0001^{*}$ \\
\hline
\end{tabular}

\begin{tabular}{|c|c|c|c|c|c|c|c|c|c|c|c|c|c|}
\cline { 2 - 13 } \multicolumn{1}{c|}{ Reader 2 } & Mean & Sdev & Variation & $P$ Value & CV & Variation & $P$ Value & Amplitude & Variation & $P$ Value & Max & Variation & $P$ Value \\
\hline CTCA $\left(^{\circ}\right)$ & 18.0 & 2.8 & $-31.2 \%$ & 0.1245 & $14.5 \%$ & $-59.7 \%$ & $0.0001^{*}$ & 7.5 & $-65.0 \%$ & $0.0001^{*}$ & 22.0 & $-39.4 \%$ & $0.0004^{*}$ \\
\hline ATCA $\left(^{\circ}\right)$ & 15.9 & 2.3 & $7.5 \%$ & 0.5404 & $14.9 \%$ & $-67.4 \%$ & $0.0000^{*}$ & 5.9 & $-65.1 \%$ & $0.0002 *$ & 18.6 & $-15.2 \%$ & 0.2754 \\
\hline MFG (mm) & 6.1 & 0.6 & $-17.5 \%$ & 0.0475 & $9.1 \%$ & $-54.8 \%$ & $0.0011^{*}$ & 1.5 & $-59.2 \%$ & $0.0010^{*}$ & 6.8 & $-21.9 \%$ & 0.0172 \\
\hline PCFU (mm) & 8.6 & 1.0 & $-89.5 \%$ & $0.0000^{*}$ & $11.3 \%$ & $-0.8 \%$ & 0.4114 & 2.8 & $-41.6 \%$ & 0.0354 & 10.1 & $-46.7 \%$ & $0.000 *^{*}$ \\
\hline
\end{tabular}

* Statistically significant $P$ values

CTCA $=$ Coronal talocalcaneal angle

ATCA $=$ Axial talocalcaneal angle

MFG = Medial facet gap

PCFU $=$ Posterior calcaneal facet uncovering 
Table 3 - Diagnostic performance of the statistically significant parameters evaluated in patients with subtalar joint stiffness using healthy volunteer data as the standard of reference.

\section{SUBTALAR JOINT STIFFNESS}

\begin{tabular}{|c|c|c|c|c|c|}
\hline \multirow[t]{2}{*}{ ATCA } & \multicolumn{2}{|c|}{ Reader 1} & \multicolumn{2}{|c|}{ Reader 2} & \multirow[b]{2}{*}{ cut off } \\
\hline & Specificity & Sensitivity & Specificity & Sensitivity & \\
\hline Max & $61 \%$ & $85 \%$ & $85 \%$ & $84 \%$ & $19.4^{\circ}$ \\
\hline CV & $72 \%$ & $85 \%$ & $89 \%$ & $85 \%$ & $31 \%$ \\
\hline Amplitude & $94 \%$ & $85 \%$ & $78 \%$ & $77 \%$ & $13.1^{\circ}$ \\
\hline \multirow[t]{2}{*}{ CTCA } & \multicolumn{2}{|c|}{ Reader 1} & \multicolumn{2}{|c|}{ Reader 2} & \\
\hline & Specificity & Sensitivity & Specificity & Sensitivity & cut off \\
\hline Max & $72 \%$ & $85 \%$ & $72 \%$ & $85 \%$ & $28.8^{\circ}$ \\
\hline $\mathrm{CV}$ & $94 \%$ & $85 \%$ & $89 \%$ & $85 \%$ & $28 \%$ \\
\hline Amplitude & $72 \%$ & $92 \%$ & $72 \%$ & $92 \%$ & $11.5^{\circ}$ \\
\hline \multirow[t]{2}{*}{ PCFU } & \multicolumn{2}{|c|}{ Reader 1} & \multicolumn{2}{|c|}{ Reader 2} & \\
\hline & Specificity & Sensitivity & Specificity & Sensitivity & cut off \\
\hline Max & $78 \%$ & $92 \%$ & $100 \%$ & $92 \%$ & $17 \mathrm{~mm}$ \\
\hline CV & $50 \%$ & $92 \%$ & $50 \%$ & $92 \%$ & $6 \%$ \\
\hline Amplitude & $50 \%$ & $100 \%$ & $50 \%$ & $100 \%$ & $1.6 \mathrm{~mm}$ \\
\hline \multirow[t]{2}{*}{ MFG } & \multicolumn{2}{|c|}{ Reader 1} & \multicolumn{2}{|c|}{ Reader 2} & \\
\hline & Specificity & Sensitivity & Specificity & Sensitivity & cut off \\
\hline Max & $56 \%$ & $62 \%$ & $83 \%$ & $62 \%$ & $8.8 \mathrm{~mm}$ \\
\hline $\mathrm{CV}$ & $72 \%$ & $100 \%$ & $61 \%$ & $100 \%$ & $9 \%$ \\
\hline Amplitude & $72 \%$ & $85 \%$ & $72 \%$ & $85 \%$ & $2 \mathrm{~mm}$ \\
\hline
\end{tabular}

CTCA = Coronal talocalcaneal angle

ATCA = Axial talocalcaneal angle

MFG = Medial facet gap

PCFU = Posterior calcaneal facet uncovering

$\mathrm{CV}=$ Variation coefficient

Max = maximum value 
Table 4 - Mean values of all parameters evaluated in patients with chronic ankle instability and variation with respect to healthy volunteers.

\begin{tabular}{|c|c|c|c|c|c|c|c|c|c|c|c|c|c|}
\cline { 2 - 13 } \multicolumn{1}{c|}{ Reader 1 } & Mean & Sdev & Variation & p Value & CV & Variation & $p$ Value & Amplitude & Variation & $p$ Value & Max & Variation & $p$ Value \\
\hline CTCA $\left(^{\circ}\right)$ & 23.7 & 6.4 & $0.3 \%$ & 0.879 & $29.1 \%$ & $-23.7 \%$ & 0.115 & 16.8 & $-27.7 \%$ & 0.148 & 32.4 & $-11.7 \%$ & 0.410 \\
\hline ATCA $\left(^{\circ}\right)$ & 13.1 & 5.2 & $-12.9 \%$ & 0.049 & $47.4 \%$ & $3.3 \%$ & 0.446 & 13.2 & $-28.7 \%$ & 0.04 & 19.3 & $-13.4 \%$ & 0.036 \\
\hline MFG (mm) & 9.3 & 0.9 & $23.4 \%$ & 0.016 & $9.9 \%$ & $-50.7 \%$ & 0.015 & 2.3 & $-61.3 \%$ & 0.067 & 10.5 & $16.6 \%$ & 0.047 \\
\hline PCFU (mm) & 12.5 & 2.0 & $-30.6 \%$ & 0.006 & $16.2 \%$ & $29.4 \%$ & 0.313 & 5.1 & $8.3 \%$ & 0.733 & 15.2 & $-25.2 \%$ & $0.001 *$ \\
\hline
\end{tabular}

\begin{tabular}{|c|c|c|c|c|c|c|c|c|c|c|c|c|c|}
\cline { 2 - 12 } \multicolumn{1}{c|}{ Reader 2 } & Mean & Sdev & Variation & $p$ Value & CV & Variation & $p$ Value & Amplitude & Variation & $p$ Value & Max & Variation & $p$ Value \\
\hline CTCA $\left(^{\circ}\right)$ & 20.46 & 5.79 & $-15.42 \%$ & 0.166 & $29.2 \%$ & $-23.1 \%$ & 0.343 & 15.1 & $-41.7 \%$ & 0.036 & 28.2 & $-28.8 \%$ & 0.015 \\
\hline ATCA $\left(^{\circ}\right)$ & 17.47 & 5.68 & $15.51 \%$ & 0.976 & $37.6 \%$ & $-21.7 \%$ & 0.563 & 14.7 & $-16.1 \%$ & 0.420 & 24.6 & $11.0 \%$ & 0.832 \\
\hline MFG (mm) & 6.76 & 1.16 & $-5.28 \%$ & 0.756 & $19.0 \%$ & $-5.6 \%$ & 0.605 & 3.0 & $-24.2 \%$ & 0.321 & 8.3 & $-5.7 \%$ & 0.598 \\
\hline PCFU (mm) & 10.17 & 2.16 & $-60.36 \%$ & $0.000 *$ & $22.1 \%$ & $48.4 \%$ & 0.006 & 5.6 & $16.0 \%$ & 0.128 & 13.2 & $-44.3 \%$ & $0.000^{*}$ \\
\hline
\end{tabular}

* Statistically significant $P$ values

CTCA $=$ Coronal talocalcaneal angle

ATCA $=$ Axial talocalcaneal angle

MFG = Medial facet gap

PCFU $=$ Posterior calcaneal facet uncovering 


\section{Figure legends}

Fig. 1 - Series of photographs demonstrating patient positioning and motion in a pronosupination cycle. Images 1, 2 and 3 demonstrate subtalar joint pronation, neutral position and supination respectively. The gray elastic band is used to guide motion throughout the prono-supination cycle.

Fig. 2 - Position of the reference markers for the four measurements evaluated. All markers were placed on the multiplanar reformats on the cortical bone. A) Coronal image at the bi malleolar plane showing the coronal talocalcaneal angle (CTCA): Two makers were placed on the medial and lateral extremes of the talar dome (line 1) and two were placed at the lateral most-point of the sustentaculum tali and another on deepest portion of Gissagne's angle (line 2). The angle between these two lines represented the CTCA. B) Axial images showing the axial talocalcaneal angle (ATCA): One marker was placed on the summit of the talar head and another on the postero-medial process of the talus (Stieda process) (line 1). At the level of the calcaneus, one marker was placed in the middle of the calcaneocuboid articular facet and another in the middle of the posterior calcaneal surface. The angle between these two lines represented the ATCA. C) Medial sagittal image showing the medial facet gap (MFG) measuring procedure: markers were placed on the narrowest point between the medial facets of the talus and the calcaneus. The distance between these two markers represented the MFG. D) Sagittal image going through the center of Gissagne's angle showing the posterior calcaneal facet uncovering (PCFU). The markers were place at the extremes of the largest distance between the center of Gissagne's angle and the anterior border of the posterior facet of the talus. The distance between these two markers represented the PCFU. 
Fig. 3 - Graphic demonstrating the ratio variation of the coronal talocalcaneal angle (CTCA), axial talocalcaneal angle (ATCA), medial facet gap (MFG) and posterior calcaneal facet uncovering (PCFU) in a 25 year-old female during prono-supination of the subtalar joint.

Fig. 4-66 year-old male with a history of proximal tibial fracture treated surgically presenting ankle pain and subtalar joint stiffness. A) Anteroposterior radiograph of the right leg depicting a chronic comminutive tibial fracture (arrow) and a transverse mid shaft fibular fracture (arrowhead). B) Sagittal CT imaging demonstrating signs of diffuse bone demineralization with mottled areas of bone resorption suggesting the diagnosis of type I complex regional pain syndrome. C) Graphic demonstrating the ratio variation of the coronal talocalcaneal angle (CTCA), axial talocalcaneal angle (ATCA), medial facet gap (MFG) and posterior calcaneal facet uncovering (PCFU). Note the global decrease in joint amplitude compared to normal subjects.

Fig. 5- 25 year-old female with a history of multiple ankle sprains and persistent ankle pain. A) Axial T2-weighted fat-saturated MR image of the ankle demonstrating a loss of fiber definition and signal hyperintensity of the anterior talofibular ligament. particularly adjacent to the fibular insertion (arrow). Graphic demonstrating the ratio variation of the coronal talocalcaneal angle (CTCA), axial talocalcaneal angle (ATCA), medial facet gap (MFG) and posterior calcaneal facet uncovering (PCFU). Despite similar motion amplitudes with respect to healthy volunteers there is a change in curve morphology of unclear significance. 
Supplementary material 1 - Lateral view of a 4D volume rendered CT images of 66 year-old male with subtalar joint stiffness.

Supplementary material 2 - Lateral view of a 4D volume rendered CT images of 25 year-old female with chronic ankle instability. 


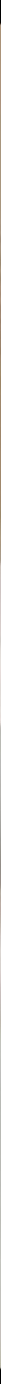



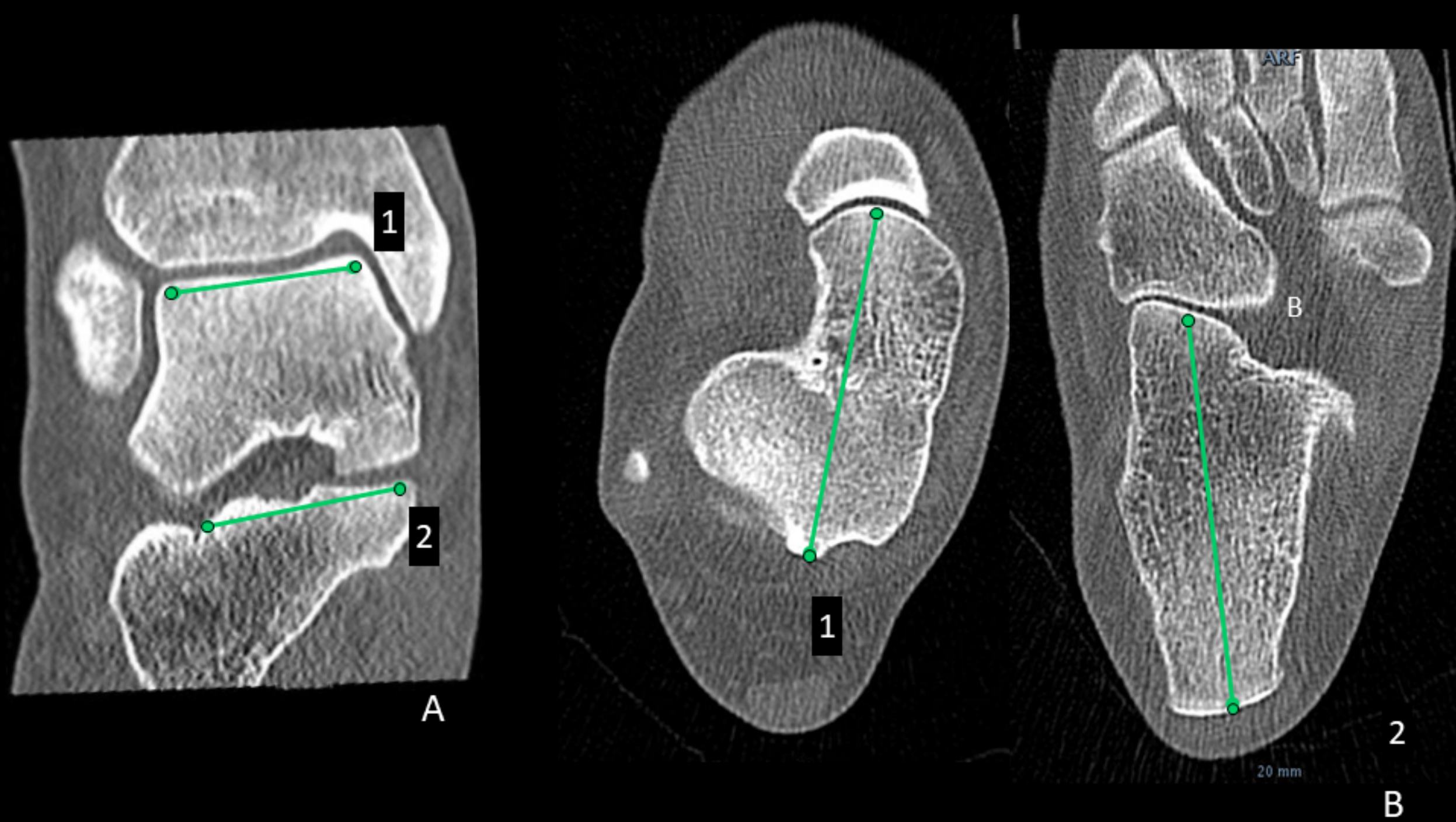

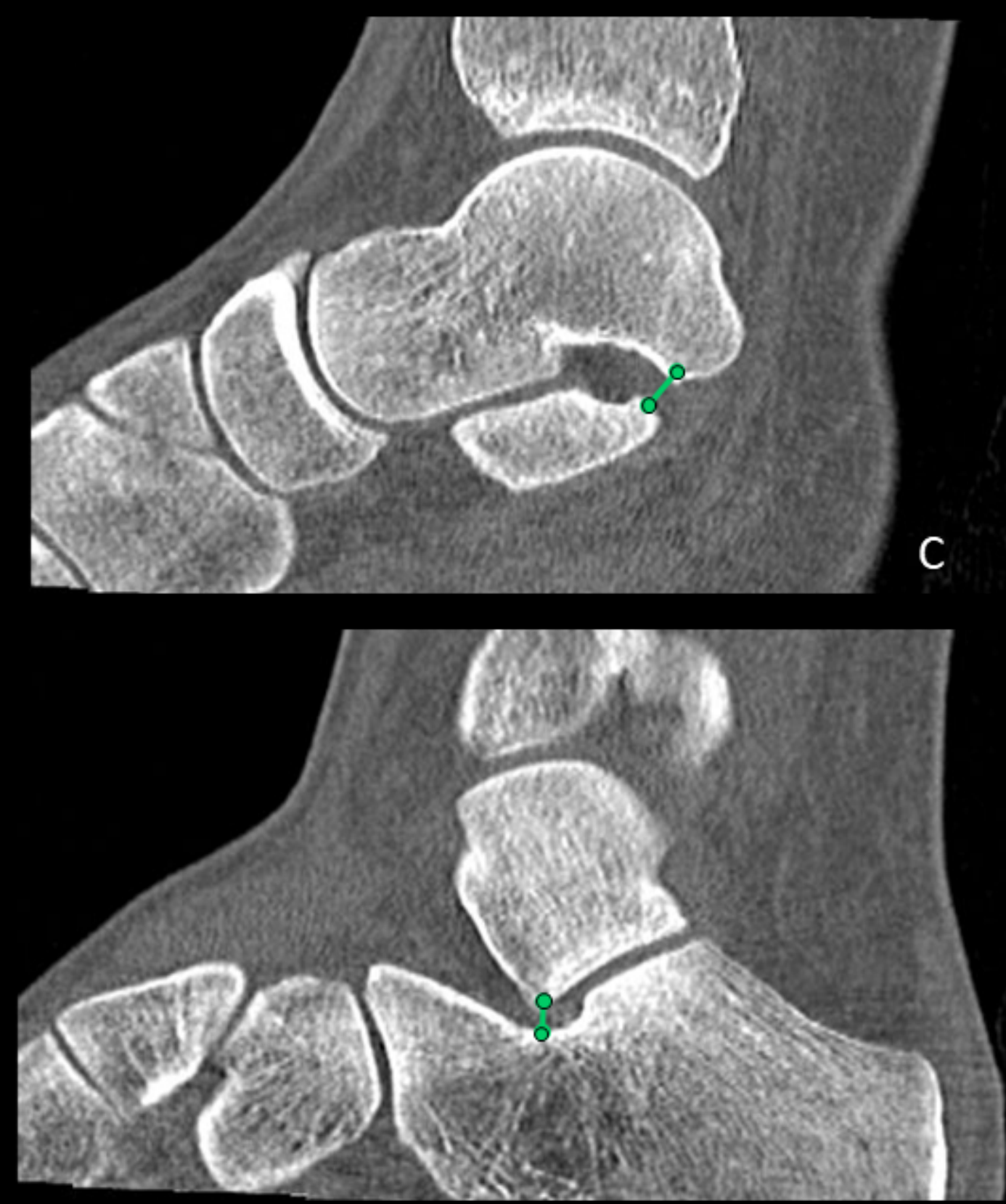

D 


\section{Ratio}

\section{Healthy volunteer}

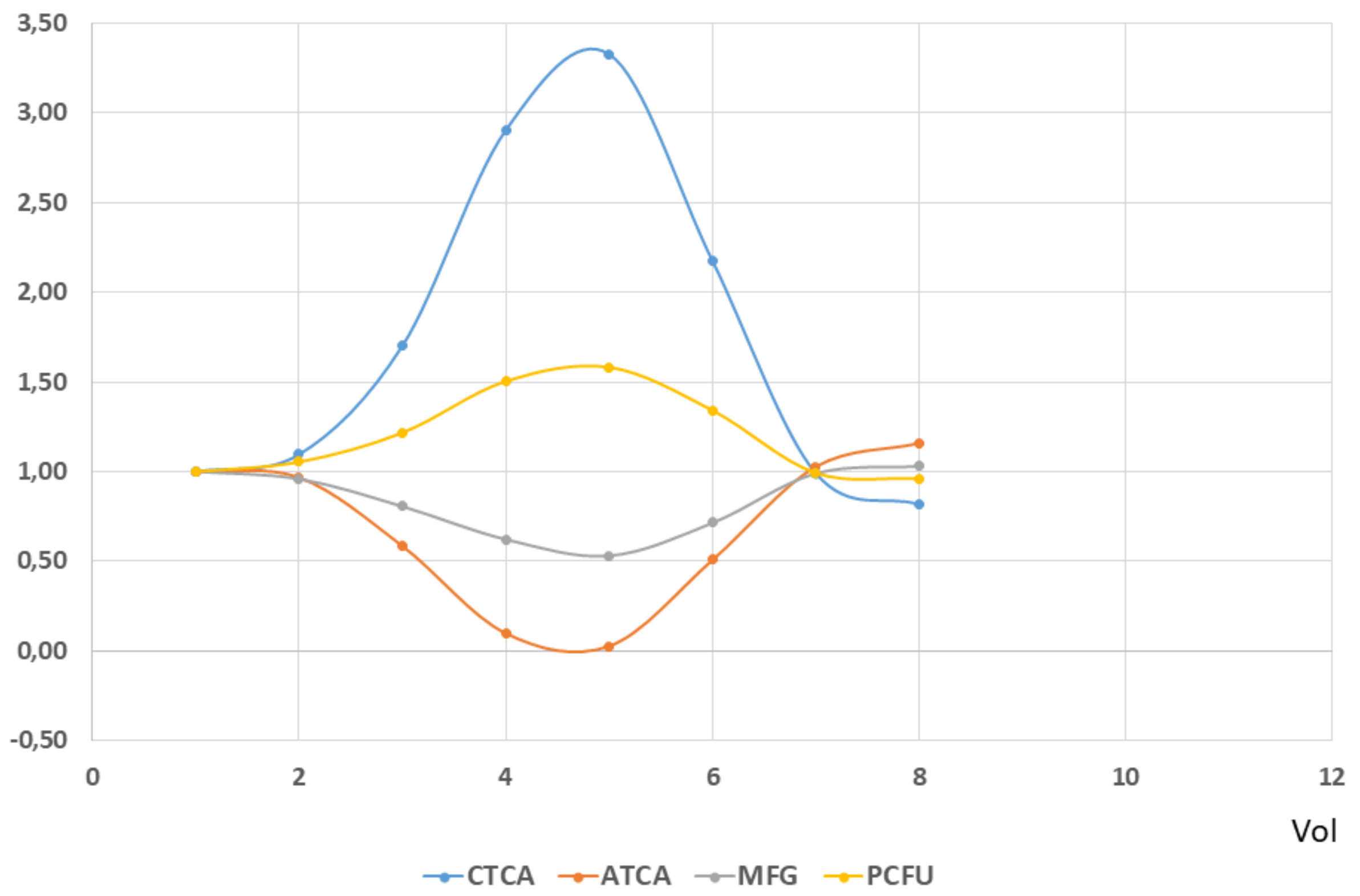




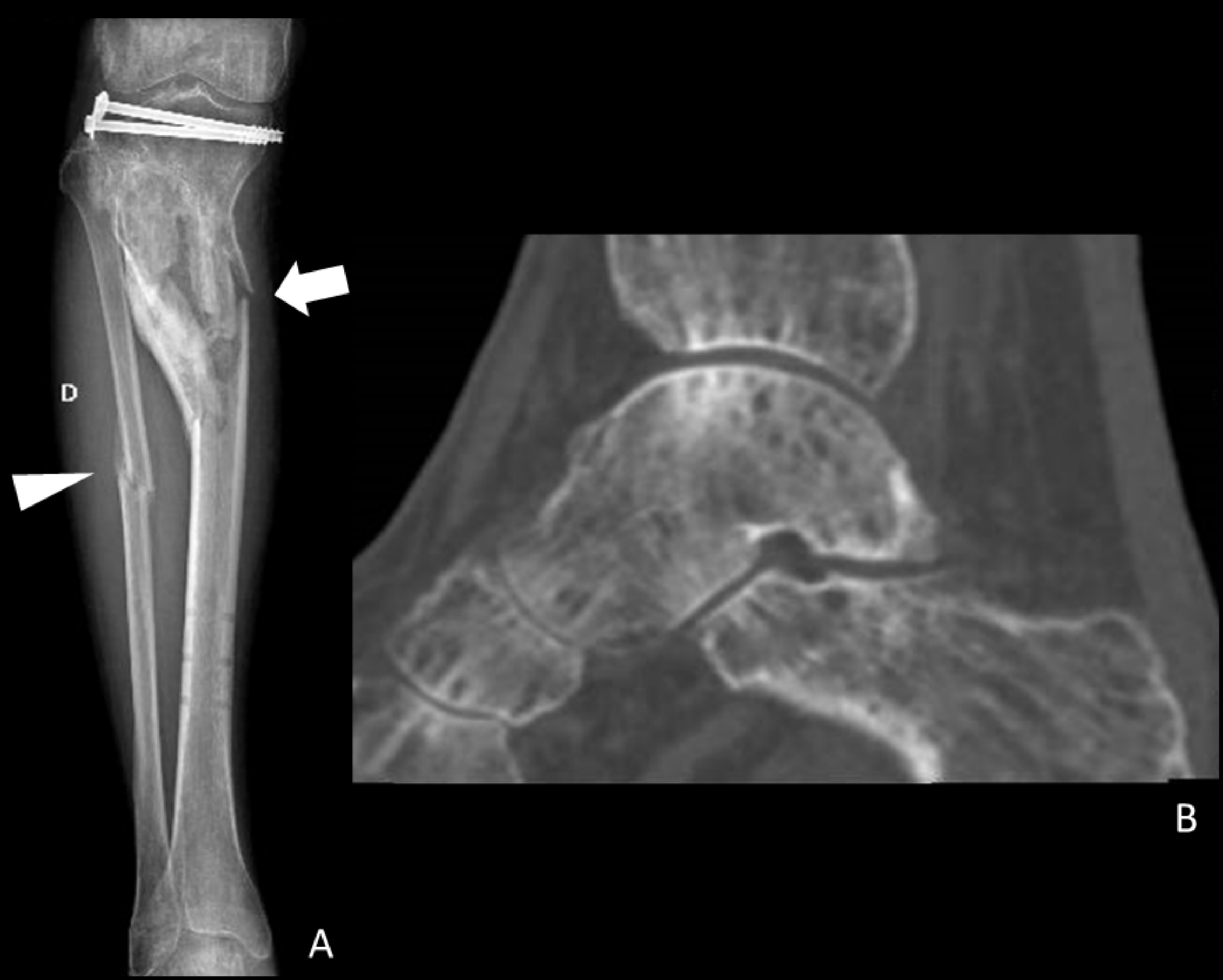




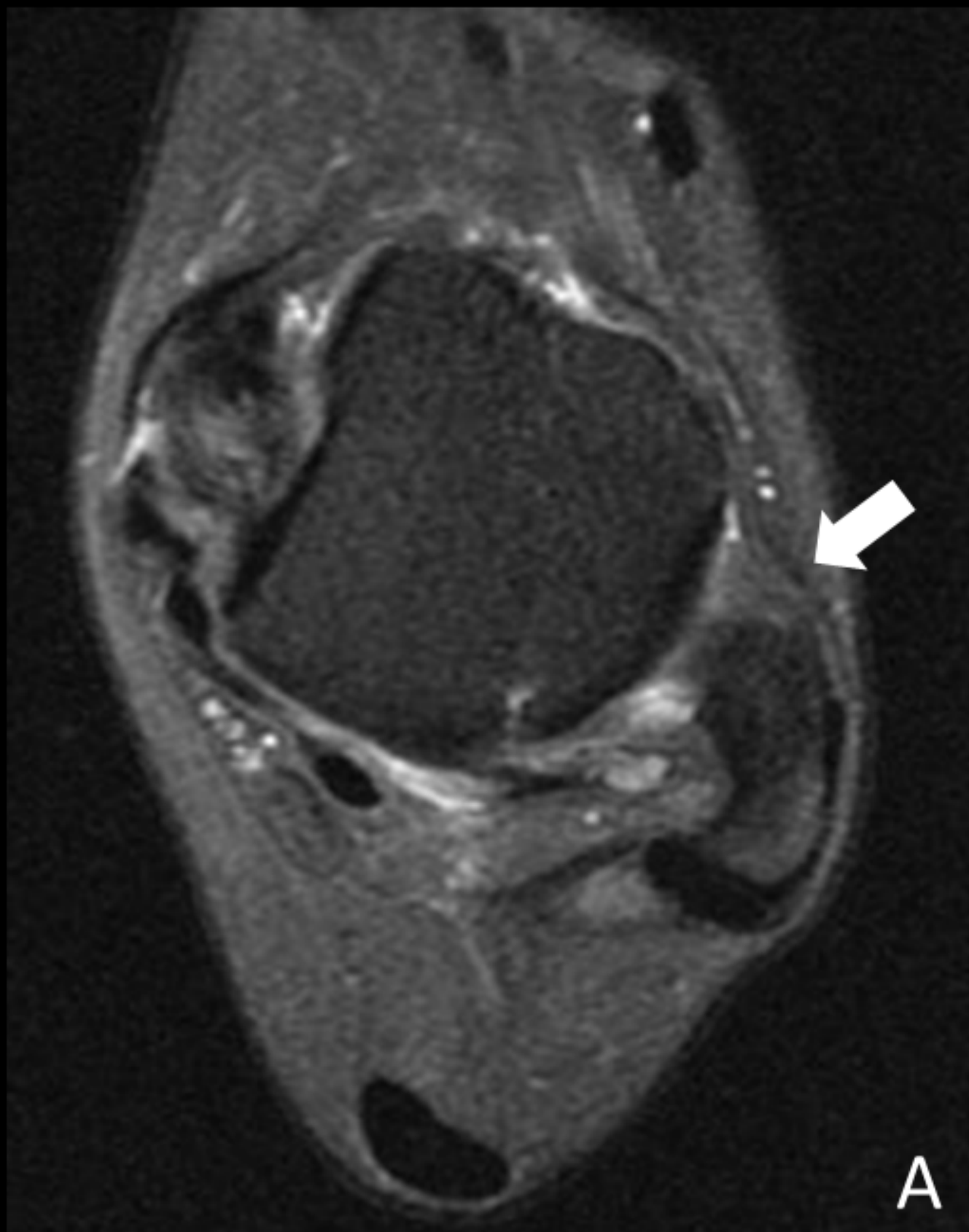




\section{Chronic ankle instability}

\section{Ratio}

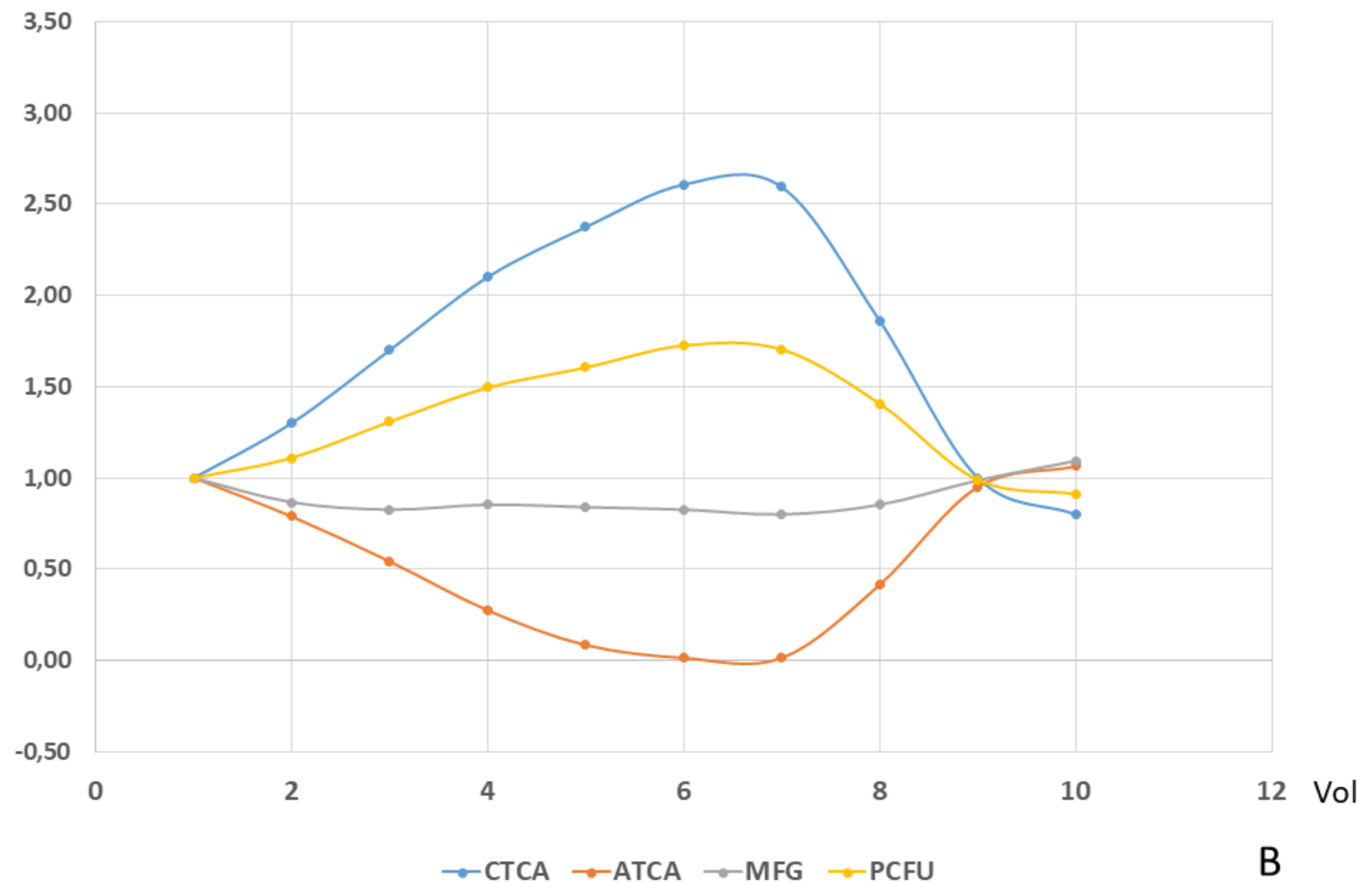

